\title{
Triazenes as a Stable Diazonium Source for Use in Functionalizing Carbon Nanotubes in Aqueous Suspensions-Supporting Information
}

\author{
Jared L. Hudson, Huahua Jian, Ashley D. Leonard, Jason J. Stephenson and James M. Tour*
}

Departments of Chemistry and Mechanical Engineering and Materials Science, and Center for Nanoscale Science and Technology, Rice University, MS 222, 6100 Main Street, Houston, Texas 77005

tour@rice.edu

4-Chlorophenyl (3,3-diethyl)triazene (2a). 4-Chloroaniline (10.00 g, 78.4 mmol) was dissolved in dichloromethane $(100 \mathrm{~mL})$ in a dry $250 \mathrm{~mL}$ round bottom flask equipped with a stir bar under an atmosphere of $\mathrm{N}_{2}$. The reaction vessel was then placed in an ice bath and the solution was stirred. To the solution was then added via syringe $\mathrm{BF}_{3} \cdot \mathrm{Et}_{2} \mathrm{O}$ (22.28 $\left.\mathrm{g}, 157 \mathrm{mmol}\right)$, followed by slow addition of isoamyl nitrite $(14.64 \mathrm{~g}, 104 \mathrm{mmol})$ over $5 \mathrm{~min}$. This mixture was stirred at 0 ${ }^{\circ} \mathrm{C}$ for $1 \mathrm{~h}$ during which time a solid formed in the flask. $\mathrm{K}_{2} \mathrm{CO}_{3}(32.48 \mathrm{~g}, 235 \mathrm{mmol})$ was added followed by slow addition of diethylamine $(17.19 \mathrm{~g}, 235 \mathrm{mmol})$ by syringe over $2 \mathrm{~min}$. A mild exothermic reaction ensued and the solid dissolved. This mixture was stirred at room temperature for $1 \mathrm{~h}$ to ensure complete conversion to the triazene. The resultant mixture was diluted with dichloromethane and extracted with $\mathrm{H}_{2} \mathrm{O}(100 \mathrm{~mL})$ three times, the organic solution was concentrated in vacuo, and the residue purified by column chromatography (alkaline silica gel, hexanes $\left./ \mathrm{CH}_{2} \mathrm{Cl}_{2}=3 / 1\right)$ to afford 2a $(13.60 \mathrm{~g}, 94 \%)$ as a red oil. IR (neat): 2975 (m), 1486$1007(\mathrm{~s}) \mathrm{cm}^{-1} ;{ }^{1} \mathrm{H} \mathrm{NMR}\left(\mathrm{CDCl}_{3}, 400 \mathrm{MHz}\right) \delta 7.37(\mathrm{~d}, J=8.8 \mathrm{~Hz}, 2 \mathrm{H}), 7.29(\mathrm{~d}, J=8.8 \mathrm{~Hz}, 2 \mathrm{H})$, $3.76(\mathrm{q}, J=7.2 \mathrm{~Hz}, 4 \mathrm{H}), 1.28(\mathrm{t}, J=7.0,6 \mathrm{H}) ;{ }^{13} \mathrm{C} \mathrm{NMR}\left(\mathrm{CDCl}_{3}, 100 \mathrm{MHz}\right) \delta 149.8,130.1$, 128.8, 121.6; HRMS calcd for $\mathrm{C}_{10} \mathrm{H}_{14} \mathrm{ClN}_{3} 211.087625$, found 211.088080. 
4-Chlorophenyl SWNT (2b). A procedure similar to the formation of $\mathbf{1 b}$ using $2 \mathbf{a}(0.059 \mathrm{~g}$, $0.32 \mathrm{mmol})$, and an aqueous suspension of SWNTs $(25 \mathrm{~mL}, 40 \mathrm{mg} / \mathrm{L}, 0.08 \mathrm{meq} \mathrm{C})$ was carried out to yield 2b $(0.002 \mathrm{~g})$. Raman D/G ratio 0.72; XPS atomic concentration C 97.98, N 0.98, Cl 1.04; TGA mass loss $17 \%$.

4-Iodophenyl (3,3-diethyl)triazene (3a). ${ }^{1}$ To a mixture of $\mathrm{K}_{2} \mathrm{CO}_{3}(6.62 \mathrm{~g}, 48.0 \mathrm{mmol})$, diethylamine ( $3.3 \mathrm{~mL}, 32 \mathrm{mmol})$, and $\mathrm{H}_{2} \mathrm{O}(40 \mathrm{~mL})$, was added a solution of 4-iododiazobenzene tetrafluoroborate ${ }^{2}(5.10 \mathrm{~g}, 16.0 \mathrm{mmol})$ in $\mathrm{CH}_{3} \mathrm{CN}(100 \mathrm{~mL})$. The reaction mixture was stirred in an ice bath that was allowed to warm to room temperature overnight. The reaction mixture was poured into water in a separatory funnel and extracted with EtOAc. The organic layer was washed with $\mathrm{H}_{2} \mathrm{O}$ twice, brine once, concentrated in vacuo and purified by column chromatography (hexanes/ $\left.\mathrm{CH}_{2} \mathrm{Cl}_{2}=3 / 1\right)$ to afford 3a $(4.75 \mathrm{~g}, 98 \%)$ as an orange oil.; ${ }^{1} \mathrm{H}$ NMR $\left(\mathrm{CDCl}_{3}, 400 \mathrm{MHz}\right) \delta 7.68(\mathrm{~d}, J=8.6 \mathrm{~Hz}, 2 \mathrm{H}), 7.24(\mathrm{~d}, J=8.6 \mathrm{~Hz}, 2 \mathrm{H}), 3.77(\mathrm{q}, J=7.1 \mathrm{~Hz}, 4 \mathrm{H})$, $1.30($ br t $, J=7.1 \mathrm{~Hz}, 6 \mathrm{H}) ;{ }^{13} \mathrm{C} \mathrm{NMR}\left(\mathrm{CDCl}_{3}, 100 \mathrm{MHz}\right) \delta 151.4,138.2,123.0,89.5$.

4-Iodophenyl SWNT (3b). A procedure similar to the formation of $\mathbf{1 b}$ using $\mathbf{3 a}(0.097 \mathrm{~g}, 0.32$ mmol), and an aqueous suspension of SWNTs ( $25 \mathrm{~mL}, 40 \mathrm{mg} / \mathrm{L}, 0.08 \mathrm{meq} \mathrm{C})$ was carried out to yield $3 \mathbf{b}(0.002 \mathrm{~g})$. Raman D/G ratio 0.65; XPS atomic concentration C 98.91, N 0.83, I 0.27; TGA mass loss $16 \%$.

4-tert-Butylphenyl (3,3-diethyl)triazene (4a). 4-tert-Butyldiazobenzene tetrafluoroborate (1.00 g, $4.0 \mathrm{mmol}$ ) was dissolved in $25 \mathrm{~mL} \mathrm{CH}_{3} \mathrm{CN}$ in a dry $50 \mathrm{~mL}$ round bottom flask equipped with a stir bar under an atmosphere of $\mathrm{N}_{2}$. To this was added $\mathrm{K}_{2} \mathrm{CO}_{3}(1.11 \mathrm{~g}, 8.0 \mathrm{mmol})$ followed by slow addition of diethylamine $(0.585 \mathrm{~g}, 8.0 \mathrm{mmol})$. The reaction mixture was allowed to stir for $1 \mathrm{~h}$ at which point it was diluted with $\mathrm{CH}_{2} \mathrm{Cl}_{2}(50 \mathrm{~mL})$ and washed with $\mathrm{H}_{2} \mathrm{O}$ 
$(50 \mathrm{~mL})$ three times. The organic solution was concentrated in vacuo, and the residue purified by column chromatography (alkaline silica gel, hexanes $\left./ \mathrm{CH}_{2} \mathrm{Cl}_{2}=3 / 1\right)$ to afford $4 \mathbf{a}(0.780 \mathrm{~g}$, 84\%) as a red oil. IR (neat): 2966 (s), 1503-1102 (m) $\mathrm{cm}^{-1} ;{ }^{1} \mathrm{H}$ NMR $\left(\mathrm{CDCl}_{3}, 400 \mathrm{MHz}\right) \delta 7.44$ (m, 4H), 3.81 (q, $J=7.2 \mathrm{~Hz}, 4 \mathrm{H}), 1.41(\mathrm{~s}, 9 \mathrm{H}), 1.32(\mathrm{t}, J=7.2 \mathrm{~Hz}, 6 \mathrm{H}) ;{ }^{13} \mathrm{C} \mathrm{NMR}\left(\mathrm{CDCl}_{3}, 100\right.$ MHz) $\delta 148.9,148.0,127.9,125.6,34.4,31.4$; HRMS calcd for $\mathrm{C}_{14} \mathrm{H}_{23} \mathrm{~N}_{3}$ 233.1892, found 233.1898.

4-tert-Butylphenyl SWNT (4b). A procedure similar to the formation of $\mathbf{1 b}$ using $4 \mathbf{a}(0.079 \mathrm{~g}$, $0.32 \mathrm{mmol})$, and an aqueous suspension of SWNTs (50 mL, $40 \mathrm{mg} / \mathrm{L}, 0.16 \mathrm{meq} \mathrm{C})$ was carried out to yield $4 \mathbf{b}(0.004 \mathrm{~g})$. Raman D/G ratio 0.42 ; XPS atomic concentration C 99.28, N 0.72; TGA mass loss $49 \%$.

Methyl 4-(3,3-diethyl)triazenebenzoate (5a). Methyl 4-diazobenzoate tetrafluoroborate ${ }^{2}$ (1.00 g, $4 \mathrm{mmol}$ ) was dissolved in $25 \mathrm{~mL} \mathrm{CH}_{3} \mathrm{CN}$ in a dry $50 \mathrm{~mL}$ round bottom flask equipped with a stir bar under an atmosphere of $\mathrm{N}_{2}$. To this was added $\mathrm{K}_{2} \mathrm{CO}_{3}(1.11 \mathrm{~g}, 8 \mathrm{mmol})$ followed by slow addition of diethylamine $(0.585 \mathrm{~g}, 8 \mathrm{mmol})$. The reaction mixture was allowed to stir for $1 \mathrm{~h}$ at which point it was diluted with $\mathrm{CH}_{2} \mathrm{Cl}_{2}(50 \mathrm{~mL})$ and washed with $\mathrm{H}_{2} \mathrm{O}(50 \mathrm{~mL})$ three times. The organic solution was concentrated in vacuo to afford $\mathbf{5 a}(0.960 \mathrm{~g}, 99 \%)$ as a red oil. IR (neat): 2978 (w), 1713 (s), 1601 (m), 1436-1099 (m) $\mathrm{cm}^{-1} ;{ }^{1} \mathrm{H}$ NMR $\left(\mathrm{CDCl}_{3}, 400 \mathrm{MHz}\right) \delta$ $7.97(\mathrm{~d}, J=8.8 \mathrm{~Hz}, 2 \mathrm{H}), 7.41(\mathrm{~d}, J=8.8 \mathrm{~Hz} 2 \mathrm{H}), 3.85(\mathrm{~s}, 3 \mathrm{H}), 3.73(\mathrm{q}, J=7.2 \mathrm{~Hz}, 4 \mathrm{H}), 1.22(\mathrm{~m}$, $6 \mathrm{H}) ;{ }^{13} \mathrm{C} \mathrm{NMR}\left(\mathrm{CDCl}_{3}, 100 \mathrm{MHz}\right) \delta 167.2,154.9,130.6,126.2,120.1,51.9$; HRMS calcd for $\mathrm{C}_{12} \mathrm{H}_{17} \mathrm{~N}_{3} \mathrm{O}_{2} 235.1321$, found 235.1317.

Methyl 4-benzoate SWNT (5b). A procedure similar to the formation of $\mathbf{1 b}$ using $5 \mathbf{a}(0.075 \mathrm{~g}$, $0.32 \mathrm{mmol})$, and an aqueous suspension of SWNTs $(50 \mathrm{~mL}, 40 \mathrm{mg} / \mathrm{L}, 0.16 \mathrm{meq} \mathrm{C})$ was carried 
out to yield $5 \mathbf{b}(0.003 \mathrm{~g})$. Raman $\mathrm{D} / \mathrm{G}$ ratio 0.56 ; XPS atomic concentration $\mathrm{C} 78.74, \mathrm{~N} 1.03, \mathrm{O}$ 20.23; TGA mass loss $9 \%$.

4-(2-Hydroxyethyl)phenyl(3,3-diethyl)triazene (6a). $\mathrm{NOBF}_{4}(3.06 \mathrm{~g}, 26.2 \mathrm{mmol})$ was added to a dry $100 \mathrm{~mL}$ round bottom flask equipped with a stir bar under an $\mathrm{N}_{2}$ atmosphere. To this was added $25 \mathrm{~mL}$ of $\mathrm{CH}_{3} \mathrm{CN}$ followed by slow addition of a slurry of 4-aminophenethyl alcohol (3.00 g, $21.9 \mathrm{mmol}$ ) in $25 \mathrm{~mL}$ of $\mathrm{CH}_{3} \mathrm{CN}$ at $0{ }^{\circ} \mathrm{C}$. The mixture was allowed to stir for $1 \mathrm{~h}$ at which point $\mathrm{K}_{2} \mathrm{CO}_{3}(6.05 \mathrm{~g}$, $43.8 \mathrm{mmol})$ was added followed by slow addition of diethylamine (3.20 g, $43.8 \mathrm{mmol})$ at $0{ }^{\circ} \mathrm{C}$. After stirring for $1 \mathrm{~h}$ the solution was filtered to remove excess $\mathrm{K}_{2} \mathrm{CO}_{3}$ followed by removal of $\mathrm{CH}_{3} \mathrm{CN}$ via rotary evaporation. The residue was dissolved in $\mathrm{CH}_{2} \mathrm{Cl}_{2}(100 \mathrm{~mL})$ and washed with $100 \mathrm{~mL}$ portions of $\mathrm{H}_{2} \mathrm{O}$, brine, $\mathrm{NaHCO}_{3}$, and a final wash of $\mathrm{H}_{2} \mathrm{O}$. The organic layer was concentrated in vacuo to afford $6 \mathbf{a}(4.39 \mathrm{~g}, 91 \%)$ as a red oil. IR (neat): 2977 (s), 1503-1043 (s) $\mathrm{cm}^{-1} ;{ }^{1} \mathrm{H} \mathrm{NMR}\left(\mathrm{CDCl}_{3}, 400 \mathrm{MHz}\right) \delta 7.34(\mathrm{~d}, J=8.4 \mathrm{~Hz}, 2 \mathrm{H})$, $7.14(\mathrm{~d}, J=8.5 \mathrm{~Hz}, 2 \mathrm{H}), 3.74(\mathrm{t}, J=6.8 \mathrm{~Hz}, 2 \mathrm{H}), 3.71(\mathrm{q}, J=7.2 \mathrm{~Hz}, 4 \mathrm{H}), 2.78(\mathrm{t}, J=6.8 \mathrm{~Hz}$, 2H), $2.45(\mathrm{~s}, 1 \mathrm{H}), 1.22(\mathrm{t}, J=7.2 \mathrm{~Hz}, 6 \mathrm{H}) ;{ }^{13} \mathrm{C} \mathrm{NMR}\left(\mathrm{CDCl}_{3}, 100 \mathrm{MHz}\right) \delta 149.8,135.3,129.4$, 120.5, 63.6, 38.8; HRMS calcd for $\mathrm{C}_{12} \mathrm{H}_{19} \mathrm{~N}_{3} \mathrm{O} 221.1528$, found 221.1528.

4-(2-Hydroxyethyl)phenyl SWNT (6b). A procedure similar to the formation of 1b using 6a (0.620 g, $2.8 \mathrm{mmol})$, and an aqueous suspension of SWNTs (200 mL, $40 \mathrm{mg} / \mathrm{L}, 0.7 \mathrm{meq} \mathrm{C})$ was carried out to yield $6 \mathbf{b}(0.012 \mathrm{~g})$. Raman D/G ratio 0.52 ; XPS atomic concentration $\mathrm{C} 83.70, \mathrm{~N}$ 1.55, O 14.75; TGA mass loss 39\%.

2-[2-(4-Nitrophenoxy)ethyl]isoindole-1,3-dione (7). 4-Nitrophenol (3.64 g, $26.2 \mathrm{mmol}), 2$ hydroxyethylphthalimide $(5.00 \mathrm{~g}, 26.2 \mathrm{mmol})$, and dicyclohexylcarbodimide $(5.40 \mathrm{~g}, 26.2 \mathrm{mmol})$ were added to a dry $250 \mathrm{~mL}$ round bottom flask equipped with a stir bar. The mixture was 
allowed to stir under a $\mathrm{N}_{2}$ atmosphere at $125{ }^{\circ} \mathrm{C}$ for $12 \mathrm{~h}$. Dichloromethane was added to the resulting solid and insoluble dicyclohexylurea was removed by filtration. The supernatant was concentrated under reduced pressure and the crude reaction material was purified by silica chromatography (alkaline silica gel, $\left.\mathrm{CH}_{2} \mathrm{Cl}_{2}\right)$ to afford $7(4.09 \mathrm{~g}, 50 \%)$ as a white powder. The product could also be recrystalized, in high purity as white needles, from ethyl acetate. IR (neat): 2921 (s), 2854 (m), 1777 (m), 1711 (s), 1606 (m), 1592 (m), 1506 (m), 1468 (m), 1427 (m), 1394 (m), 1342 (m), 1264 (m), 1173 (w), 1111 (w), 1014 (w), 843 (m), 722 (m) cm ${ }^{-1}$; ${ }^{1} \mathrm{H}$ NMR (CDCl, $400 \mathrm{MHz}) \delta 8.17(\mathrm{~d}, J=9.3 \mathrm{~Hz}, 2 \mathrm{H}), 7.86(\mathrm{~m}, 2 \mathrm{H}), 7.76(\mathrm{~m}, 2 \mathrm{H}), 6.95(\mathrm{~d}, J=$ $9.3 \mathrm{~Hz}, 2 \mathrm{H}), 4.34(\mathrm{t}, J=5.7 \mathrm{~Hz}, 2 \mathrm{H}), 4.15(\mathrm{t}, J=5.8 \mathrm{~Hz}, 2 \mathrm{H}) ;{ }^{13} \mathrm{C} \mathrm{NMR}\left(\mathrm{CDCl}_{3}, 100 \mathrm{MHz}\right) \delta$ $168.3,163.4,142.1,134.4,132.1,126.1,123.7,114.8,65.6,37.1$.

2-[2-(4-Aminophenoxy)ethyl]isoindole-1,3-dione (8). 7 (30.00 g, $96.1 \mathrm{mmol})$ was dissolved in ethyl acetate $(1 \mathrm{~L})$ in a $2 \mathrm{~L}$ Erlenmeyer flask equipped with a stir bar. The solution was then transferred in $150 \mathrm{~mL}$ portions to a $300 \mathrm{~mL}$ screw top flask containing palladium on activated carbon (10\% Pd, $0.5 \mathrm{~g})$. The flask was sealed and placed under a $\mathrm{H}_{2}$ atmosphere (50 psi) for $1 \mathrm{~h}$ at room temperature. This process was repeated on the remaining solution of 7 . The catalyst was then removed by filtration over celite and the filtrate concentrated under reduced pressure to afford 8 (26.95 g, 99 \%). IR (neat): 3440 (w), 3359 (w), 2946 (w), 1770 (m), 1712 (s), 1511 (s), 1465 (m), 1423 (m), 1394 (s), 1360 (m), 1231 (s), 1038 (m), 1023 (m), 835 (m), 718 (s) cm ${ }^{-1} ;{ }^{1} \mathrm{H}$ NMR (CDCl $3,400 \mathrm{MHz}) \delta 7.86(\mathrm{~m}, 2 \mathrm{H}), 7.72(\mathrm{~m}, 2 \mathrm{H}), 6.72(\mathrm{~d}, J=8.9 \mathrm{~Hz}, 2 \mathrm{H}), 6.60(\mathrm{~d}, J=$ $8.9 \mathrm{~Hz}, 2 \mathrm{H}), 4.15(\mathrm{t}, J=6.2 \mathrm{~Hz}, 2 \mathrm{H}), 4.06(\mathrm{t}, J=5.9 \mathrm{~Hz}, 2 \mathrm{H}), 3.41(\mathrm{br} \mathrm{s}, 2 \mathrm{H}) ;{ }^{13} \mathrm{C} \mathrm{NMR}$ $\left(\mathrm{CDCl}_{3}, 100 \mathrm{MHz}\right) \delta 168.4,151.7,140.6,134.2,132.3,123.5,116.5,116.1,65.7,37.7$.

2-[2-(4-(3,3-diethyl)triazenylphenoxy)ethyl]isoindole-1,3-dione (9). 8 (12.50 g, 44.3 mmol) was dissolved in dichloromethane $(125 \mathrm{~mL})$ in a $250 \mathrm{~mL}$ round bottom flask equipped with a stir 
bar. The reaction vessel was placed in a cold bath $\left(-78{ }^{\circ} \mathrm{C}\right)$ and kept under a $\mathrm{N}_{2}$ atmosphere. To this was added $\mathrm{BF}_{3} \bullet \mathrm{Et}_{2} \mathrm{O}(12.57 \mathrm{~g}, 88.6 \mathrm{mmol})$ via syringe over $10 \mathrm{~min}$. Isoamyl nitrite $(10.38 \mathrm{~g}, 88.6 \mathrm{mmol})$ was then added via syringe over $10 \mathrm{~min}$. The reaction mixture was then allowed to warm to room temperature at which point solid $\mathrm{K}_{2} \mathrm{CO}_{3}(18.38 \mathrm{~g}, 133 \mathrm{mmol})$ was added. The reaction was then returned to the cold bath $\left(-78^{\circ} \mathrm{C}\right)$ followed by addition of $\mathrm{Et}_{2} \mathrm{NH}$ (9.73 g, $133 \mathrm{mmol})$ via syringe over $5 \mathrm{~min}$. After the final addition the reaction vessel was removed from the cold bath and allowed to stir at room temperature for $10 \mathrm{~h}$. The mixture was then diluted with dichloromethane $(150 \mathrm{~mL})$ and washed three times with $\mathrm{H}_{2} \mathrm{O}(250 \mathrm{~mL})$. The organic layer was then dried over $\mathrm{MgSO}_{4}$, filtered and the filtrate was concentrated under reduced pressure. The crude product was purified by silica chromatography (alkaline silica gel, $\left.\mathrm{CH}_{2} \mathrm{Cl}_{2} / \mathrm{Hex}=1 / 1\right)$ to afford 9 (10.35 g, 64 \%). IR (neat): $2975(\mathrm{w}), 2940(\mathrm{w}), 2876(\mathrm{w}), 1774$ (m), 1713 (s), 1602 (w), 1504 (m), 1463 (m), 1393 (s), 1354 (m), 1240 (s), 1198 (m), 1098 (m), 1018 (m), 920 (w), 841 (w), 715 (m) cm ${ }^{-1} ;{ }^{1} \mathrm{H}$ NMR $\left(\mathrm{CDCl}_{3}, 400 \mathrm{MHz}\right) \delta 7.85$ (m, $\left.2 \mathrm{H}\right), 7.71(\mathrm{~m}$, $2 \mathrm{H}), 7.31(\mathrm{~d}, J=8.9 \mathrm{~Hz}, 2 \mathrm{H}), 6.84(\mathrm{~d}, J=8.9 \mathrm{~Hz}, 2 \mathrm{H}), 4.22(\mathrm{t}, J=5.6 \mathrm{~Hz}, 2 \mathrm{H}), 4.10$ (t, $J=5.9$ $\mathrm{Hz}, 2 \mathrm{H}) 3.71(\mathrm{q}, J=6.6 \mathrm{~Hz}, 4 \mathrm{H}), 1.23(\mathrm{t}, J=7.2 \mathrm{~Hz}, 6 \mathrm{H}) ;{ }^{13} \mathrm{C} \mathrm{NMR}\left(\mathrm{CDCl}_{3}, 100 \mathrm{MHz}\right) \delta$ $168.3,156.2,145.6,134.1,132.2,123.5,121.4,115.0,65.1,37.5$.

\section{References}

1. Jian, H; Tour, J. M. J. Org. Chem. 2005, 70, 3400.

2. Bahr J. L.; Yang J.; Kosynkin D. V.; Bronikowski M. J.; Smalley R. E.; Tour J. M. J. Am. Chem. Soc. 2001, 27, 6536. 